\title{
THE
}

\section{Spin Diffusion in Classical Heisenberg Magnets with Uniform, Alternating, and Random Exchange}

\author{
Niraj Srivastava \\ University of Rhode Island \\ Jian-Min Liu \\ University of Rhode Island \\ V. S. Viswanath \\ University of Rhode Island \\ Gerhard Müller \\ University of Rhode Island, gmuller@uri.edu
}

Follow this and additional works at: https://digitalcommons.uri.edu/phys_facpubs

Terms of Use

All rights reserved under copyright.

\section{Citation/Publisher Attribution}

Niraj Srivastava, Jian-Min Liu, V. S. Viswanath and Gerhard Müller. Spin diffusion in classical Heisenberg magnets with uniform, alternating, and random exchange. J. Appl. Phys. 75 (1994), 6751-6753.

Available at: http://dx.doi.org/10.1063/1.356842

This Article is brought to you for free and open access by the Physics at DigitalCommons@URI. It has been accepted for inclusion in Physics Faculty Publications by an authorized administrator of DigitalCommons@URI. For more information, please contact digitalcommons-group@uri.edu. 


\title{
Spin diffusion in classical Heisenberg magnets with uniform, alternating, and random exchange
}

\author{
Niraj Srivastava, a) Jian-Min Liu, V. S. Viswanath, and Gerhard Müller \\ Department of Physics, The University of Rhode Island, Kingston, Rhode Island 02881-0817
}

\begin{abstract}
We have carried out an extensive simulation study for the spin autocorrelation function at $T=\infty$ of the one-dimensional classical Heisenberg model with four different types of isotropic bilinear nearest-neighbor coupling: uniform exchange, alternating exchange, and two kinds of random exchange. For the long-time tails of all but one case, the simulation data seem incompatible with the simple $\sim t^{-1 / 2}$ leading term predicted by spin diffusion phenomenology.
\end{abstract}

The anomalous character of spin diffusion in the onedimensional (1D) classical Heisenberg model,

$$
H=-\sum_{i} J_{i, i+1} \mathbf{S}_{i} \cdot \mathbf{S}_{i+1},
$$

with uniform exchange, $J_{i, i+1}=J$, was first proposed in 1988 on the basis of a simulation study. ${ }^{1}$ The proposition was that the spin autocorrelation function at $T=\infty$ exhibits a distinctive power-law long-time tail,

$$
C_{0}(t) \equiv\left\langle\mathbf{S}_{i}(t) \cdot \mathbf{S}_{i}\right\rangle \sim t^{-\alpha},
$$

with a characteristic exponent $\alpha$ that exceeds the value $\alpha_{\mathrm{SD}}=1 / 2$ predicted by spin diffusion phenomenology considerably.

That conclusion was challenged by Gerling and Landau ${ }^{2}$ soon after it had appeared in print. However, the consensus emerged that the slope $\bar{\alpha}$ inferred from the simulation data (in a $\log -\log$ representation) bas a value $\bar{\alpha}=0.60$ at $J i \simeq 25$ and a decreasing trend for longer $J t$, and that the true asymptotic behavior remained out of the reach of simulation data available at the time., 2,3

It was nevertheless another simulation study that yielded new insights into the anomalous transport mechanism of spin fluctuations in the classical Heisenberg chain. Having been alerted by the puzzling results for the spin autocorrelation function, Bonfim and Reiter ${ }^{4}$ focused their own investigation on the $q$-dependent spin correlation functions and the associated current correlation function. One of their conclusions was that the asymptotic behavior of the spin autocorrelation function is of the form

$$
C_{0}(t) \sim[J t \ln (\Omega t)]^{-\alpha}
$$

with $\alpha=0.472$. The implication is that the slope of $C_{0}(t)$ in a $\log -\log$ plot can be described by an effective exponent,

$$
\bar{\alpha}=\alpha[1+1 / \ln (\Omega t)] \text {. }
$$

Böhm, Gerling, and Leschke ${ }^{5}$ were quick to point out that the asymptotic form of the $q$-dependent correlation function used in Ref. 4 is in contradiction to the non-negativity of $\left\langle\mathbf{S}_{i}(t) \cdot \mathbf{S}_{i+n}\right\rangle$, for which strong numerical evidence exists. Theyproposed an alternative asymptotic expression, which also implies an effective exponent (4), but with asymptotic value $\alpha=0.5$.

\footnotetext{
s:Present address: Thinking Machines Corporation, Cambridge, MA 021421264.
}

Since neither of the two conclusions was primarily based on the analysis of spin autocorrelation functions, we wish to use our own new simulation data for $C_{0}(t)$ as a discriminant between expressions (4) with $\alpha=0.472$ and $\alpha=0.5$, respectively. We have carried out the simulation for a system of 1024 spins with periodic boundary conditions. We have employed CM-5 machines with various numbers of processors programmed in Connection Machine FORTRAN for up to 4096 parallel time integrations. For the integration over the time interval $0 \leqslant J t \leqslant 102.2$, we have used a fourth-order Runge-Kutta method with fixed time step $J d t=0.005$. In this massively data-parallel programming mode we can reach previously unattained statistics with no undue effort.

For the intended analysis, we have determined the average slope $\bar{\alpha}$ of the simulation data in a $\log -\log$ representation over a time interval of length $J t_{\mathrm{ay}}$ as follows: each data point of $\tilde{\alpha}(t)$ is calculated by linear regression from $N_{\mathrm{av}}$ consecutive data points $\left[\ln J i, \ln C_{0}(l)\right]$ spaced at $J \Delta l=0.2$ and assigned the $J t$ value at the midpoint of the interval of length $J t_{\mathrm{itv}}=N_{\mathrm{av}} J \Delta t$. Figure 1 shows the slope function $\tilde{\alpha}$ plotted versus $1 / J t$ for three different sizes of $J t_{\mathrm{av}} .^{6}$ This representation enhances the visibility of the subtle features in the long-time tail, but it also magnifies the statistical fluctuations. The latter are kept under control by adjusting $J t_{\mathrm{av}}{ }^{7}$

In order to facilitate a direct comparison of our simulation results with the proposed functional form (4) for the effective exponent $\bar{\alpha}(t)$, we have subjected the asymptotic expression $\sim[J t \ln (\Omega t)]^{-\alpha}$ to the same exponent analysis as the data. The resulting slope function $\bar{\alpha}(t)$ still depends on the parameters $\alpha$ and $\Omega$. There is no compelling reason for setting $\Omega$ equal to $J$ in the logarithmic correction as was done in Refs. 4 and 5 . Minimizing the relative rms deviation between the two slope functions $\tilde{\alpha}(t)$, namely the one representing the simulation data and the one representing the averaged exponent $\tilde{\alpha}(t)$ over the interval $5+J t_{\mathrm{av}} / 2 \leqslant J t$ $\leqslant 102.2-J t_{\mathrm{av}} / 2$, yields parameter values in the range $\alpha=0.478 \pm 0.001, \Omega / J=2.30 \pm 0.02$ for the three values of averaging intervals $J t_{\mathrm{av}}$ used. The solid lines represent $\tilde{\alpha}$ vs $1 / J t$ for the optimal parameter values. The agreement with the simulation data is quite satisfactory. If we perform the fit for fixed $\alpha=0.5$, we obtain the optimal value $\Omega / J=9.70$ \pm 0.05 for the other parameter, and the result, represented by the dashed lines, is in clear disagreement with the simulation data.

We have repeated the analysis with more of the (evidently nonasymptotic) data at small times omitted (up to 


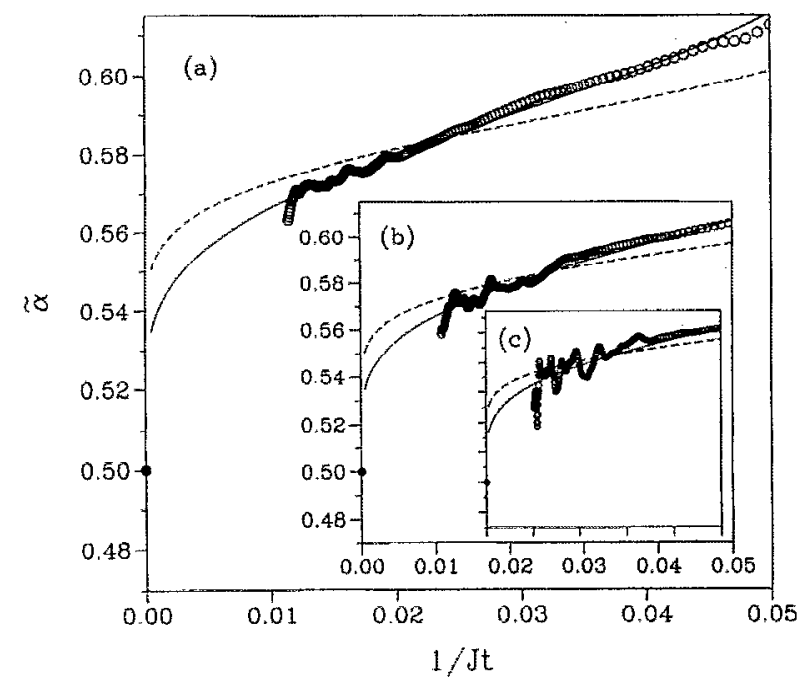

FIG. 1. Slope function $\alpha(t)$ for the $1 \mathrm{D}$ classical Heisenberg model (1) with uniform exchange, $J_{i, i+1}=J$ as determined from the slope of $C_{n l}(t)$ in a $\log -\log$ plot. The data for $C_{0}(t)$ represent an average over 404484 randomly chosen initial conditions and over the 1024 sites of the lattice. Each data point $\dot{\alpha}$ is determined by linear regression from $N_{\text {av }}$ consecutive data points $\left[\ln J t, \ln C_{0}(t)\right]$ spaced at $J \Delta t=0.2$ and plotted vs $1 / J t$ at the midpoint of the interval of length $J t_{\mathrm{av}}=N_{\mathrm{av}} J \Delta t$. The simulation data are represented by the circles. The asymptotic form (3) subjected to the same procedure yields the dashed lines for $\alpha=0.5$ and the solid lines for $\alpha=0.478$. The three plots correspond to different sizes of averaging time interval: (a) $J t_{\mathrm{av}}=30$, (b) $J t_{\mathrm{av}}=20,(\mathrm{c}) J t_{\mathrm{dv}}=10$.

$\left.15+J t_{\mathrm{av}}\right)$ and found a decreasing trend of the optimal exponent value (now in the range $\alpha=0.472 \pm 0.002$, in even better agreement with the value proposed in Ref. 4). Nevertheless, the problems attached to this scenario, as pointed out in Ref. 5 , cannot be dismissed and suggest that the true asymptotic behavior is even more subtle.

How typical is the occurrence of anomalous long-time tails in 1D classical spin systems with isotropic exchange? It had already been noted $\mathrm{d}^{4}$ that the anomaly disappears in the presence of uniaxial anisotropy. The question is what happens if we modify the spin coupling without altering the rotational symmetry in spin space, for example, by reducing or removing the translational symmetry along the chain.

In order to investigate that question, we have carried out simulations of comparable extent on three further variants of the classical Heisenberg model (1). In addition to model (i) with uniform exchange, $J_{i, i+1}=J$, discussed previously, we consider the model (ii) with alternating exchange, $J_{i, i+1}=(-1)^{i} J$, and two models with random exchange: model (iii) has $J_{i, i+1}= \pm J$ with equal probabilities and model (iv) has $\left|J_{i, i+1}\right| \leqslant \sqrt{3} J$ with a rectangular probability distribution. ${ }^{8}$ The results of this investigation are displayed in Figs. 2-4 for models (ii)-(iv) in exactly the same representation as those of model (i) exhibited in Fig. 1. The outcome is a bit surprising. It seems that long-time tails display no less individuality than, say, pony tails. ${ }^{9}$

Among the four models considered here, the one with alternating exchange alone appears to exhibit completely normal spin diffusive behavior. Its slope function $\tilde{\alpha}$, displayed in Fig. 2, tends to extrapolate on a fairly direct path toward $\alpha_{\mathrm{SD}}=0.5$. The data invite no suspicion of any

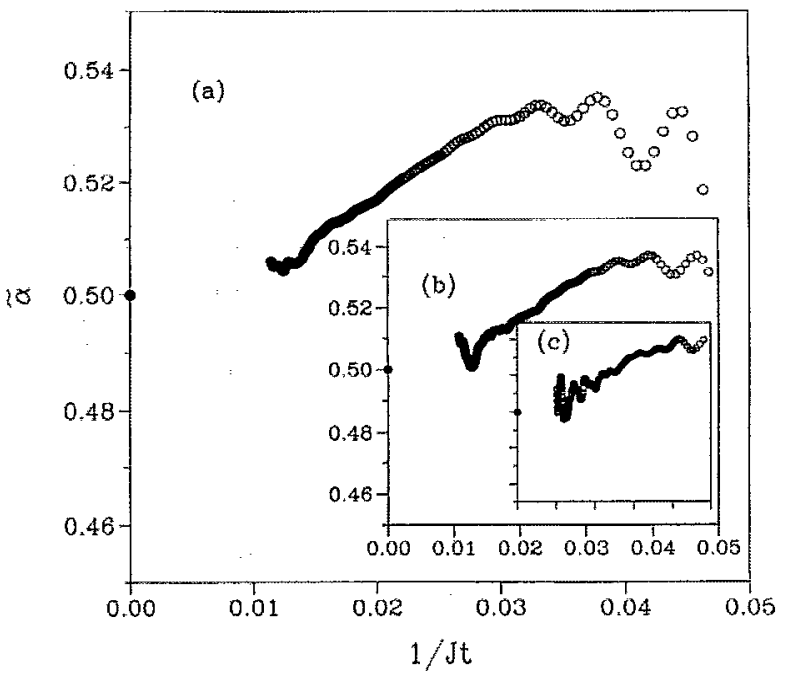

FIG. 2, Slope function $\bar{\alpha}(t)$ for the $1 \mathrm{D}$ classical Heisenberg model (1) with alternating exchange, $J_{i, i+1}=(-1)^{i} J$, produced by the same method as that of Fig. L. The number of integrations with randonly chosen initial conditions was 479232 for this case.

anomaly. The strong wiggles at small $t$ are not statistical fluctuations but originate from oscillations in $C_{00}(t)$, which persist to longer times in this model than in any of the other three.

The slope function $\tilde{\alpha}$ of the random-exchange model (iii) is shown in Fig. 3. Unlike in the previous two cases, it has an increasing trend for increasing $t$ up to $\tilde{\alpha}=0.53$ at the tail end of the data, where it seems to level off. While a limiting value of $\alpha_{\mathrm{SD}}=0.5$ cannot be ruled out, the data do not show any tendency to extrapolate to that value.

Changing the distribution of random exchange constants from (iii) to (iv) produces a quite different slope function as can be observed in Fig. 4. It starts out at a much smaller

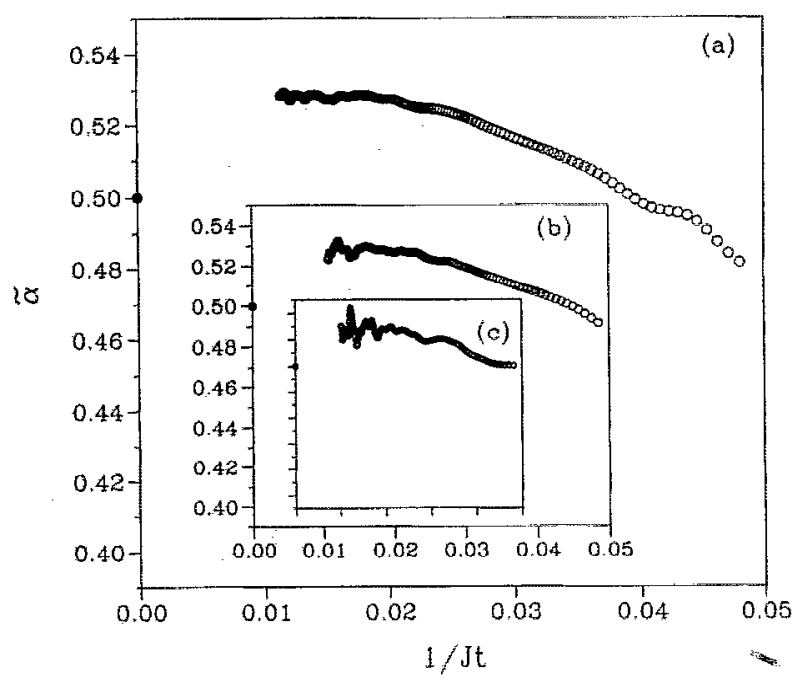

FIG. 3. Slope function $\partial(t)$ for the $1 \mathrm{D}$ classical Heisenberg model (1) with random exchange, $J_{i, i+1}= \pm J$, produced by the same method as that of Fig. 1. The number of integrations with randomly chosen initial conditions was 409600 for this case. For each initial configuration, the exchange constants were randomly chosen as well. 


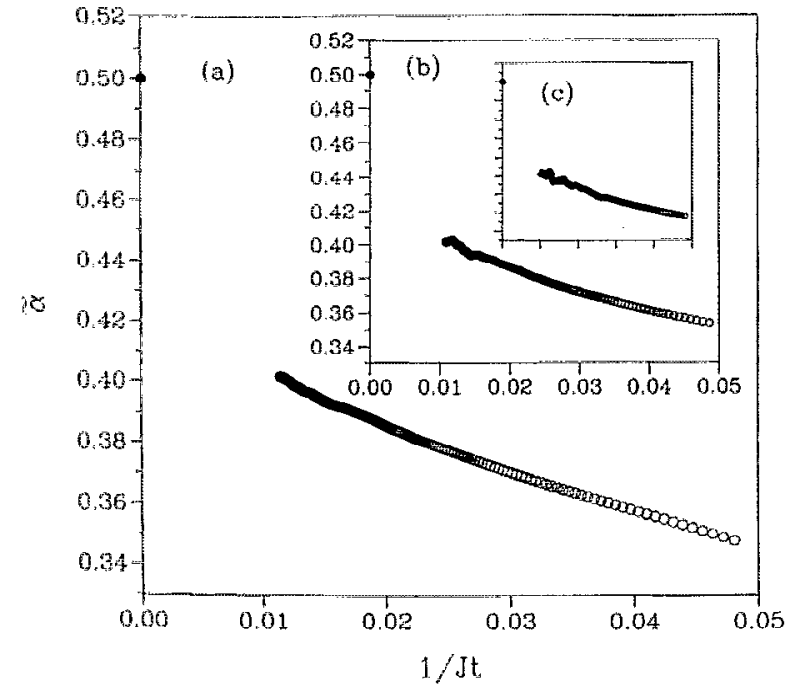

FIG. 4. Slope function $\tilde{\alpha}(t)$ for the $1 \mathrm{D}$ classical Heisenberg model (1) with random exchange, $\left|J_{i, i+1}\right| \leqslant \sqrt{3} J$, produced by the same method as that of Fig. 1. The number of integrations with randomly chosen initial condition was 424960 in this case. For each initial configuration, the exchange constants were randomly chosen as well.

value for short $t$ and reaches $\tilde{\alpha} \simeq 0.4$ at the tail end of the data $(J t=102.2)$. In some way this slope function looks like the mirror image of that for model (i). It may very well extrapolate to $\alpha_{\mathrm{SD}}=0.5$ or there abouts by some logarithmic law - a modification of Eq. (4). But any such law would have to be motivated by an investigation of $q$-dependent cor- relation functions as was done by Bonfim and Reiter ${ }^{4}$ for model (i). For the random-exchange model (iii), the anomaly, if it indeed exists, is much weaker than in models (i) and (iv) and will therefore be much harder to identify and analyze in $q$-dependent correlation functions. These are future projects.

This work was supported by the U.S. National Science Foundation, Grant No. DMR-93-12252. The simulations were run on CM-5 machines at the National Center for Supercomputing Applications, University of Illinois at UrbanaChampaign and at Thinking Machines Corporation.

'G. Müller, Phys. Rev. Lett. 60, 2785 (1988); 63, 813 (1989).

${ }^{2}$ R. W. Gerling and D. P. Landau, Phys. Rev. Lett. 63, 812 (1989); Phys. Rev. B 42, 8214 (1990).

${ }^{3}$ J.-M. Liu, N. Srivastava, V. S. Viswanath, and G. Müller, J. Appl. Phys. 70, 6181 (1991).

${ }^{4}$ O. F. de Alcantara Bunfim and G. Reiter, Phys. Rev. Lett. 69, 367 (1992); 70, 249 (1993).

${ }^{5}$ M. Böhm, R. Gerling, and H. Leschke, Phys. Rev. Lett. 70, 248 (1993).

${ }^{6}$ See Fig. 2 of Ref. 2 (second paper) and Fig. 2 of Ref. 3 for previous interpretations of slope functions based on different simulation data.

${ }^{7}$ The price to be paid in exchange of a smooth slope function is a systematic deviation from the true slope at a given value of $J t$ for all functions except pure power laws. For a given set of simulation data, if one increases $J t_{w w}$, one gains smoothness and along with it the ability to extrapolate. Conversely, if one decreases $J t_{\mathrm{av}}$, the systematic deviations go down, but the statistical fluctuations grow more intense. The three plots in cach figure are intended to illustrate that the systematic deviations are negligible except (in some cases) for short times.

"The width $2 \sqrt{3} J$ of the $J_{i, i+1}$ distribution has been chosen to match the initial curvature of $C_{0}(t)$ with those of the other three models.

${ }^{9}$ In Ref. 1 (second paper) we have already compared three of the four Iong-time tails directly and found (ii) and (iii) similar but distinct from (i). The superior statistics of our new data reveals subtle but significant differences between (ii) and (iii) as well. 